\title{
A Novel On-site Deployment, Commissioning and Debugging Technique to Assess and Validate WSN Based Smart Systems
}

\author{
Gabriel Mujica, Alejandro Garcia, Javier Gordillo, Jorge Portilla, Teresa Riesgo
}

\begin{abstract}
In this work a novel on-site toolset-based architecture for tackling the main challenges of deploying and commissioning large scale WSN-based systems is proposed. This is one of the first implementations that addresses a complete set of runtime algorithms to efficiently deploy sensor platforms in the target scenarios based on the inclusion of the real behavior of the nodes within the in-situ simulation chain, combined with the integration of runtime diagnosis and reprogramming strategies to analyze the performance of the deployment in-field.
\end{abstract}

Keywords-WSN optimization, HW-SW co-design, sensor node deployment \& commissioning.

\section{INTRODUCTION}

The Wireless Sensor Network (WSN) research field has been growing and becoming more mature during the last decade, in which novel hardware and software techniques for increasing its applicability, usability and reliability in smart scenarios have been intensively proposed by the research community. In this context, important contributions regarding the planning and modeling of wireless sensor networks have been made, targeting the theoretical optimization of node placements to find a trade-off between connectivity of nodes, coverage and hardware/deployment costs [1]. However, the effective implementation of the WSN in-situ, i.e., the real deployment and commissioning of the sensor networks in the final planned scenario presents important issues to be faced so that the final operation and the QoS of the system can be assured.

These key aspects to be considered include the transient power consumption during the installation, setup, synchronization and reprogramming steps, the time spent during the commissioning stage depending on the evaluation strategy to be adopted, and the actual placement problems that come out with the in-field WSN establishment, mainly in terms of connectivity, processing and sensing performance. Therefore, the effective combination of modelling and planning strategies with efficient deployment, commissioning and assessment techniques are to be taken into account to guarantee the final autonomy of the WSN smart system.

In order to address such a challenging approach, a novel and powerful on-site mechanism to optimize the real implementation of WSNs is proposed in this work, which integrates in a unique in-field toolset four main capabilities: an in-situ deployment connectivity matrix simulator and generator based on the real behavior of the communication modules to be used in the final deployment, to optimize the relationship between the node placement algorithm of the planning tools with respect to the actual transmission power to be dynamically configured in the final hardware platforms; a novel on-site deployment methodology based on a set of algorithms that make use of the aforementioned deployment matrix generation to propose an optimized strategy to install and validate the sensor nodes in an efficient fashion, depending on the target environment to act on; an on-site automatic localization and guidance system to help deployers to install and study in real time the performance of the nodes during the deployment process; and an over-the-air debugging system to dynamically assess the current configuration of the sensor nodes as well as reconfigure or even reprogram the platform in runtime. The main target is to be able to feed back modeling and planning tools with the in-field commissioning and performance evaluation of the sensor network within outdoor application scenarios, so that a refinement of the simulation models can be made to generate an optimized/environment-adapted deployment plan.

There are few proposals focused on efficient in-field WSN commissioning, debugging and validation techniques and even a lack of works related to deployment methodologies and strategies to maximize the time, power and resource consumption during the installation, configuration and evaluation stage of the wireless sensor nodes. In [2], a commissioning and deployment method is proposed mainly focused on a node reprogramming mechanism once they are unboxed, setting the role they shall adopt in the network deployment. A diagnosis framework for WSNs is presented in [3] to assess the performance of the sensor nodes once the network is at the operational stage, which is intended to be a general approach for designers to test and verify running applications.

\section{System ARchitecture \& Proposed Platform}

The system architecture is based on the implementation of a Hand-Held Device (HHD) through which the deployment and commissioning activities are performed in-field. The HHD integrates two main elements: a mobile device connected to a WSN-HW platform that implements the corresponding lowrate communication protocol (HHNode). In this work, the technology that is under test is the Cookies platform [4], which is a HW-SW modular node that allows including different communication layers depending on the application requirements, such as IEEE 802.15.4 or ZigBee. Based on this schema, the tool can have on-site access both to the planning/development models (by means of using a RESTful web service interface) and to the wireless nodes that are to be deployed. A general view of the proposed architecture is shown in figure 1, where the integration of the implemented subsystems is highlighted, as well as the overall functional flow of the toolset. 


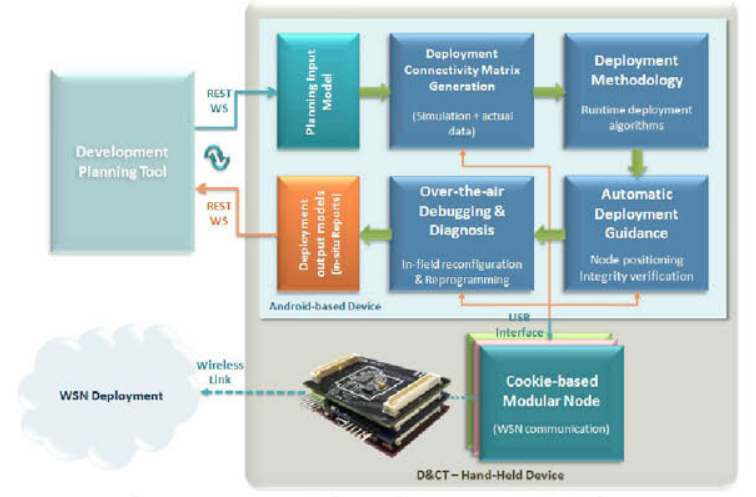

Figure 1. General view of the proposed design.

\section{A. Deployment connectivity simulation system}

In combination with the theoretical node coordinates generated from the planning tool models, an on-site deployment connectivity matrix simulator is proposed in this work to address two major goals: first, supply the deployment methodology sub-system with an experimental estimation of the node's connectivity in order to consider what nodes are correlated before executing the deployment mechanism; second, dynamically optimize the radio transmission power configuration of the nodes depending on real connectivity models generated from the actual hardware to be used in-field. Thereafter, once the deployment stage is executed, a real connectivity matrix is generated and compared to the former estimation; then the planning tool will be fed back so as to enhance the simulation models according to the gathered data from the specific conditions of the target scenario.

Three parameters will be used to carry out the simulation and generation of the connectivity matrix, i.e., RSSI, LQI and LDR, as well as the distance between pair nodes in an outdoor scenario. One of the most common models to predict the received power of wireless signals is the log-normal shadow model [5][6], which can be expressed in $\mathrm{dBm}$ as follows (1):

$$
\operatorname{RSSI}(\mathrm{dBm})(d)=\operatorname{RSSI}(\mathrm{dBm})\left(d_{0}\right)-10 \cdot n \cdot \log _{10}\left(\frac{d}{d_{0}}\right)
$$

Where $d$ is the distance between nodes $(\mathrm{m}), d_{0}$ is the reference distance $(\mathrm{m})$ and $n$ is a path-loss exponent. There are typical values for $n$ depending on the application environment [6]. However, the idea is rather to adapt the corresponding model to the specific target scenario and the HW platform to be used, based on on-site measurements from the HHD, so as to provide an estimation of the connectivity matrix according to a more accurate path-loss custom factor. Focused on this approach, experimental tests have been set up to model the Cookie platform with the included IEEE 802.15.4 based communication layer, which integrates a CC2420 module. At one meter height in an outdoor free space scenario, the distance between two pair nodes and the transmitted power used are shown in fig. 2a) b). For each distance-power combination, five tests have been performed, gathering the RSSI, LQI and LDR metrics (back and forth values), for a total of 760 tests and 4560 samples automatically launched and obtained from the HHD tool. Based on the average values taken from all the distance-power combinations, an experimental path-loss expression can be obtained from (1):

$$
n=\frac{R S S I\left(d_{0}\right)-R S S I(d)}{10 \cdot \log _{10}\left(d / d_{0}\right)}
$$

As a result, applying (2) to all the average measured values, the on-site path-loss factor $\mathbf{n}_{\mathbf{m}}=\mathbf{1 . 7 7}$. Results are then compared to the simulation model (1) by using this experimental/custom factor (e.g., fig. 2a) for Tx Power = $5 \mathrm{dBm})$. These experimental results also allow obtaining the threshold values from which two nodes can be considered as unconnected (unstable link), i.e., $\mathbf{L D R} \leq \mathbf{9 8 \%}$ and $\mathbf{R S S I}_{\text {lim }}=$ -85. This information is used by the deployment methodology system to know the whole picture of the node's correlation before applying the corresponding algorithms. Again from (1), the distance limit can be expressed as follows (3):

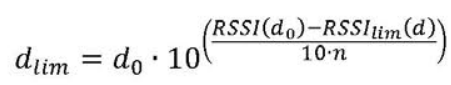

Applying to (3) the optimized path-loss factor and based on all the TX powers, the limit distance results are shown in fig. 2b). Hence, based on the input model coordinates, the TX power of the HW platform can be dynamically configured insitu so as to optimize the power consumption of the nodes in relation with their distance/RSSI correlation.

\section{B. Deployment methodology and strategies}

In this work a novel on-site deployment methodology is proposed based on a set of mathematical tools and algorithms adapted to the deployment optimization problem, by defining a cost function that represents the amount of energy/resources spent in deploying the nodes within the target scenario, which has to be minimized. First of all, it is necessary to remark that WSNs can be deployed mainly following two approaches: by deploying nodes one after the other while checking their connectivity to the rest of the surrounding nodes (even if their theoretical neighbours are not set yet); or considering that the nodes are placed in their corresponding locations (based on a pre-deployment/placement stage) and then the deployment tasks are performed, which reduces the number of configuration and establishment scans to be executed by the deployer as well as the network/link map generation process, since the number of effective neighbour coverage per target node is higher while the number of configuration executions is reduced. Assuming the energy consumption as the cost unit, the first method can be mathematically expressed as:

$$
f_{\text {cost }}=\sum_{i=1}^{n}\left[\frac{d(i, i-1)}{S m} \cdot P_{s} \cdot i+E_{\text {scan }}+E_{\text {conf }} \cdot \text { neigh } h_{\text {act }}(i)\right]
$$

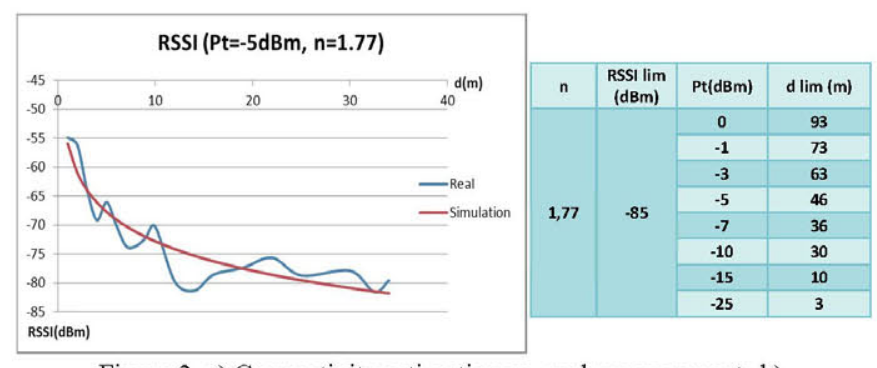

Figure 2. a) Connectivity estimation vs. real measurements b) dynamic TX Power config. based on distance/RSSI limits. 
Where $n$ is the number of nodes in the network, $S_{m}$ a distance cost factor, $P_{s}$ is the power that consumes a node in standby mode, $E_{\text {scan }}$ is the consumption spent by each node to scan its neighbours, $E_{\text {conf }}$ is the consumption spent on establishing a connection and configuration of nodes, neigh $h_{a c t}$ is the number of already deployed nodes that are directly connected to node $i$. The second method is expressed as:

$f_{\text {cost }}=\alpha * n+\sum_{i=1}^{n^{\prime}}\left[\frac{d(i, i-1)}{S m} \cdot P_{s} \cdot n+E_{\text {scan }}+E_{\text {conf }} \cdot n e i g h_{\text {act }}(i)\right]$

$n$ ' is the number of nodes that need to be configured to establish all connections and $\alpha$ is a parameter representing how much energy per node was spent on the deployment. Once these functions are defined, three mechanisms are proposed to optimize the deployment task: swap-based algorithm, genetic-based algorithm and clustering algorithm.

The swap-based algorithm (SA) could be considered as the most effective but the least efficient method. It consists on a number of permutations of an array of nodes, while analysing every possible route and returns the one with the least cost function result. Its main limitation is the time requested to get the optimum sequence: since in order to add an extra node to a network of $k$ nodes, the number of possibilities is $(k+1)$ times larger, then the number of possibilities of an $n$-size network is $n$ !. As a result, the simulation process is no longer useful for networks larger than 10 nodes, taking into account that the simulation is to be run on-site by using the HHD.

As an alternative, a genetic-algorithm-based mechanism (GA) adapted to the proposed deployment methodology is introduced. As it is well known, these iterative algorithms mimic the process of natural selection and, although they provide a sub-optimal solution, a trade-off between simulation time and efficient result generation makes this approach more suitable and adaptable for the proposed technique. The first step of the genetic algorithm is to generate a population of candidate solutions. This usually means transform a solution into binary code, although in this case, the genotype is an array of the nodes that form the candidate deployment sequence. Thereafter, each codified individual is to undergo a series of processes: selection, crossover, mutation and replacement [7], which fitness (inversely proportional to the cost result) has to be evaluated, i.e., how good that solution is. Thereby, new deployment routes will be formed with paths of the best available sequences in the population, which means that eventually, these new routes will be close to be optimal.

The clustering algorithm (CA) has been developed focusing on the robustness of the network instead of the mathematical optimum deployment strategy. This method searches for closed groups of nodes within the network in order to

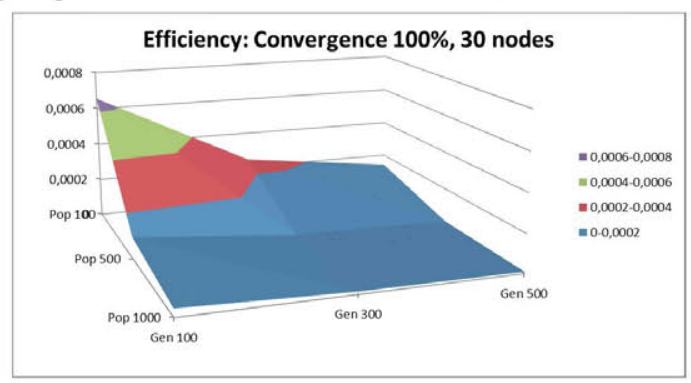

Figure 3. Efficiency of GA based on generations \& population. modularize the deployment task. The deployer has several sub-networks to deploy, which implies a considerable saving of time and energy because once a target group is debugged and validated, the following tasks are transparent for it and, thus, nodes that are part of that group do not affect the energy cost variability of the remaining process. Groups are automatically generated by closing an interconnection sequence between a reference node and the subsequent neighbours until the former one is again part of such sequence. The main parameter to be considered is the limit of nodes allowed per group, which effects strongly depend on the network distribution. Once the identification of the groups has been made, SA or GA are used to find a suitable sequence that covers the entire network passing through every group.

Several simulation test cases were set up to analyse the proposed mechanism according to the defined cost functions (based on the generation of sub-random distributions of sensor nodes and varying the number of deployed nodes, population size, generations, convergence limit rate and maximum number of nodes per group in case of CA), as well as find experimentally an optimal configuration to be used in final application scenarios. As a result, more than 700 cases were contemplated. Applying the SA for both methods (4) (5), the cost-result (5) is lower than the first one, although for 9 nodes the simulation time is considerably longer. In case of the GA, results show that the simulation time grows as population size and the limit of generation does, although the cost decreases. In order to compare both effects, a new measure was added: the efficiency, which is defined as $E_{f f}=1 / \operatorname{Cost}$.Ts. The result for the first method with no convergence limit is quite illustrating (fig. 3), which is similar for (5). This is caused by the simulation time: it grows exponentially with the increase of population and generations, while the cost is more linear.

According to the simulation, a convergence limit of $75 \%$ is too low for several cases, so the default limit will be around $90 \%$. It is also important to highlight that, for instance, considering a population size of 100 and a limit of 300 generations (a trade-off solution) as default values, in case of 30 nodes the cost obtained by the second function is no longer lower than that obtained by the first one. This is caused by the stand-by energy state of the nodes within the second method. Therefore, this parameter will be also a key factor to be considered when selecting the best/more adapted solution. In case of the CA, although there is a dependency on the network distribution, in general the second function offers solutions with a considerably lower cost result as the network gets larger. Based on these results, a comparison in terms of cost

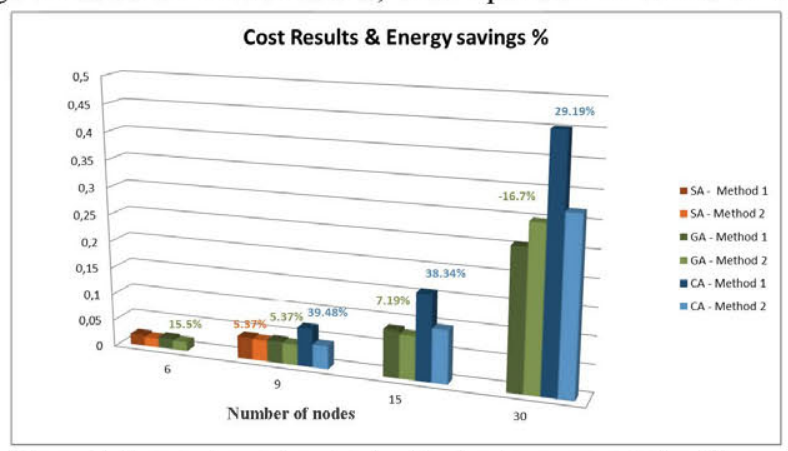

Figure 4. Comparison of cost reduction for the proposed algorithms. 


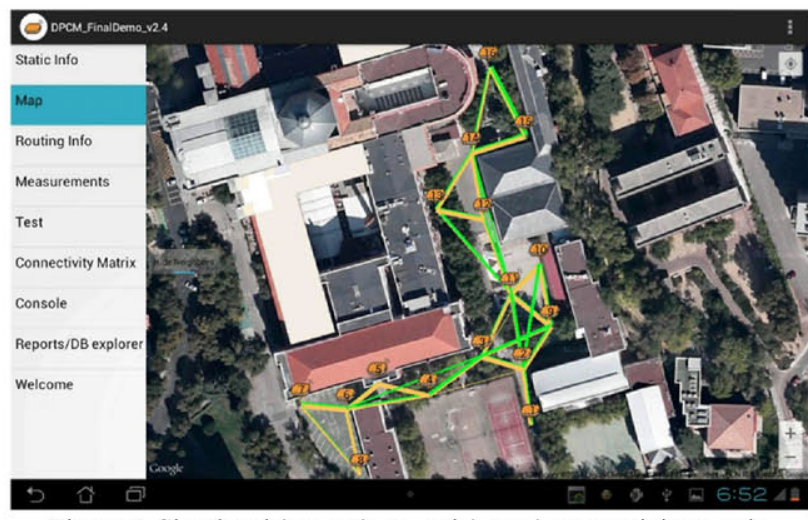

Figure 5. Simulated (orange) vs. real (green) connectivity matrix.

reduction of (5) with respect to (4) by applying the three proposed algorithms is shown in fig. 4. The GA has been set to default values and the clustering algorithm set to a limit per group of $50 \%$ of the total network. Similar results have been obtained for SA and GA, which means that the last one can provide a closely optimized solution (SA is supposed to be the best bellow 10 nodes). However, the main cost reduction is found by applying the $\mathrm{CA}$, which can be more efficient in terms of network validation from the deployer perspective.

\section{On-site Automatic Guidance \& debugging System}

After applying the aforementioned strategies and based on the node coordinates of the network model input, an in-field automatic guidance is launched by the HHD to help deployers during the installation process. By combining the GPS+Wi-Fi capabilities of the mobile device with the connectivity of the HHNode, users can be guided to the precise place where nodes shall be installed and verify its integrity by means of executing HW-SW platform tests from the debugging sub-system. This component is in charge of obtaining sensor platform parameters based on the communication of the HHD with the deployed nodes by using the wireless protocol. The sensor nodes integrate a "plug-in" to debug the HW-SW implementation on top of the IEEE 802.15.4 based on the commissioning frames transmitted from the HHD. This debugging interface also allows reprogramming specific code components of the wireless node application by using the memory-segmentation-based architecture proposed in [8].

\section{EXPERIMENTAL RESULTS \& ANALYSIS}

A real outdoor deployment has been carried out in the ETSII-UPM to verify the integration of the designed subsystems so that an on-site commissioning and debugging process is performed by using the HHD. The WSN is composed of 16 nodes which positions are downloaded from a planning tool (in a JSON file) as the input of the system. Deployers can select one of the proposed deployment algorithms and methods or let the tool automatically analyse both approaches and get the least expensive in terms of time and energy/resource consumption. The GA generated the best cost function results (with a configuration of population $=100$, generations $=300$, convergence $=90 \%$ ) for the second method (in this case the network distribution penalized the CA results) and with a deployment sequence of $15,14,12,10,11,2,3,5,6$, ended in node 7 (the remaining nodes are automatically covered and configured in such route sequence). Thereafter,
TABLE I. SIMULATED VS. REAL CONNECTIVITY. AVERAGE ERRORS

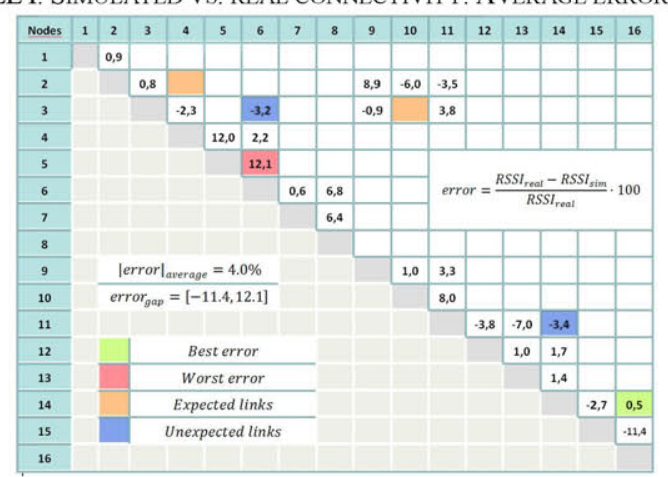

both the simulated and the real in-field connectivity matrices taken by running the HHD tool are highlighted in fig. 5 (orange and green, respectively), along with the experimental results regarding the deviation of the actual data from the estimated values (table I, which shows the error calculated per average values). The TX power has dynamically been set to $-5 \mathrm{dBm}$ according to the simulation results, and 3 consecutive tests per link were launched on-site (the width of the lines represents the quality of the link). 2 unexpected connections have been established (3-6 and 11-14, with -86.3 and -86.4 of RSSI simulated, respectively), whereas 2 expected connections have failed (2-4 and 3-10, with -84.6 and -84.1 RSSI simulated, respectively) over 24 links. Estimated vs. real values have a $4 \%$ overall average error ( 0.5 as minimum, 12.1 as maximum). In general, a positive error (the simulated value is better than the real one) can be related to obstacles and placement deviations; whereas negative errors to line-of-sight scenarios.

\section{CONCLUSIONS}

A powerful on-site HHD-based toolset has been proposed to cope with the main challenges and lack of defined methods for efficiently carrying out the deployment and commissioning of WSN based systems, so as to assure the long-term operability of the final application. Both the connectivity simulator and the set of deployment algorithms were applied to an actual in-field deployment so that the real behavior of the network is actively included in the functional chain of the system.

\section{REFERENCES}

[1] S. Misra Mi, S. D. Hong, G. Xue and Jian Tang, "Constrained Relay Node Placement in Wireless Sensor Networks: Formulation and Approximations", trans. Networking, vol. 18, no. 2, pp. 434-447, 2010.

[2] B, W, Kim and S.-S, Joo, "A New Commissioning and Deployment Method for Wireless Sensor Networks," in Proc. UBICOMM'09, pp, 232-237, October 2009.

[3] D. Yu, "DiF: A Diagnosis Framework for Wireless Sensor Networks," in Proc. INFOCOM'10, pp.1-5, San Diego, CA, 2010.

[4] J. Portilla, T. Riesgo, A. Abril, A. de Castro, "Rapid prototyping for multi-application sensor networking", 12 November 2007, SPIE Newsroom. DOI: 10.1117/2.1200711.0851.

[5] M. Botta, M. Simek, "Adaptive Distance Estimation Based on RSSI in 802.15.4 Network", in Journal of Radioingenieering, vol.2, no.4, 2013.

[6] J. Kang, D. Kim, and Y. Kim, "RSS self-calibration protocol for WSN localization," in Proc. ISWPC'07, Feb. 2007.

[7] O. Nurika, N. Zakaria, F. Hassan, and L.T. Jung, "Workability review of genetic algorithm approach in networks," in ICCOINS'14, pp.1-6, 2014.

[8] G. Mujica, V. Rosello, J. Portilla, and T Riesgo, "On-the-fly dynamic reprogramming mechanism for increasing the energy efficiency and supporting multi-experimental capabilities in WSNs," in Proc. IECON'13, pp.5455-5460, Nov. 2013. 